\title{
GAMIFICACIÓN PARA FOMENTAR LA ACTIVACIÓN DEL ALUMNADO EN SU APRENDIZAJE
}

\author{
GAMIFICATION TO IMPROVE STUDENT ACTIVATION IN LEARNING \\ GAMIFICAÇÃO PARA PROMOVER A ATIVAÇÃO DE ALUNOS EM SUA \\ APRENDIZAGEM
}

\author{
Ma Elena Parra-González \\ Universidad de Granada, Espanha \\ elenaparra@ugr.es \\ Adrián Segura-Robles \\ Universidad de Granada, Espanha \\ adrianseg@ugr.es \\ Esteban Vázquez Cano \\ Universidad Nacional de Educación a Distancia, Espanha \\ evazquez@edu.uned.es \\ Eloy López-Meneses \\ Universidad Pablo de Olavide, Espanha \\ elopmen@upo.es
}

RESUMEN: La gamificación es una de las metodologías activas actuales utilizadas para motivar al alumnado y que de manera activa construyan su aprendizaje. En esta investigación, se analiza la activación desde la perspectiva de las experiencias gamificadas en estudiantes universitarios. Dentro del conjunto de dimensiones desarrolladas y puesta a prueba por la literatura científica ponemos en valor la importancia de la misma como precursora de otro tipo de ventajas para el desarrollo educativo de los estudiantes. Los resultados indican valores altos de activación para estudiantes inmersos en actividades gamificadas además de que no existen variables sociodemográficas que afecten a este tipo de valores de forma significativa.

PALABRAS CLAVE: Educación. Gamificación. Metodología. Activación.

ABSTRACT: Gamification is one of the current active methodologies used to motivate students and actively build their learning. In this research, activation is analyzed from the perspective of gamified experiences in university students. Within the set of dimensions developed and tested by the scientific literature, we value its importance as a precursor to other advantages for the educational development of students. The results indicate high activation values for students immersed in gamified activities, in addition to the fact that there are no sociodemographic variables that affect these types of values significantly. KEYWORDS: Education. Gamification. Methodology. Activation.

RESUMO: A gamificação é uma das atuais metodologias ativas utilizadas para motivar os alunos e construir ativamente a sua aprendizagem. Nesta pesquisa, a ativação é 
analisada sob a ótica das experiências gamificadas em estudantes universitários. Dentro do conjunto de dimensões desenvolvidas e testadas pela literatura científica, valorizamos sua importância como precursora de outros tipos de vantagens para o desenvolvimento educacional dos alunos. Os resultados indicam altos valores de ativação para alunos imersos em atividades gamificadas, além do fato de não existirem variáveis sociodemográficas que afetem significativamente esse tipo de valores.

PALAVRAS-CHAVE: Educação. Gamificação. Metodologia. Ativação.

\section{Introducción}

Una de las grandes preocupaciones de la docencia hoy día es la falta de motivación (RODRÍGUEZ-PÉREZ, 2012), implicación y actividad de los estudiantes en su proceso de aprendizaje. Los docentes deben intentar que sus alumnos aprendan en clase, y mucha parte de ello, va a depender de la actitud de los mismos. En este panorama surge la necesidad de contar con distintas metodologías, técnicas y herramientas que fomenten la activación del alumnado para construir su aprendizaje. Estos cambios metodológicos normalmente no funcionan de manera independiente, sino que suelen ir asociados a toras variables. En este sentido, cabe destacar que en la actualidad la mayoría de las veces estos cambios van asociados a los avances tecnológicos, así muchas metodologías deben su nacimiento al uso de las mismas, como es el caso por ejemplo de la gamificación y el aprendizaje invertido (SEGURA-ROBLES et al., 2020).

Según (LABRADOR PIQUER; ANDREU ANDRÉS, 2008) las metodologías activas son los métodos que, incluyen también las técnicas y las estrategias, que pueden utilizar los docentes en su proceso de enseñanza y reconvertir este en actividades que permitan fomentar a los mismos la participación del estudiante para conducirles a aprender. Una de las características más representativas de estas metodologías activas es que se busca la transformación del alumnado para convertirse en agentes activos de su aprendizaje, a través de la búsqueda de su actitud activa (LIRA VALDIVIA, 2010). Por ello, las metodologías activas pueden promover y ofrecer condiciones adecuadas para que estos agentes puedan comprometerse con los procesos de enseñanza-aprendizaje (LUELMO DEL CASTILLO, 2018).

Esta renovación metodológica en cuanto a la implementación de metodologías activas en los entornos educativos, necesitará de una preparación y formación del profesorado, para ser capaces de ofrecerles al alumnado una experiencia de aprendizaje activo (FERNANDEZ-MARCH, 2006). Cuando los alumnos están activos, construyen su aprendizaje de manera significativa y son los protagonistas de su propio aprendizaje (NEVES, 2013).

Además, una de las grandes verdades universales es que el niño necesita jugar (PIAGET, 2013), y es mediante el juego como se va a relajar y crear mundos mediante los cuales se va a desarrollar (MONTAÑÉS RODRÍGUEZ et al., 2000). El juego forma parte de su vida como algo natural, y es utilizado en educación en numerosas ocasiones como una herramienta natural de aprendizaje, dados los beneficios que ello conlleva (BENITEZMURILLO, 2009; FOSTER, 2008). 
Surge así la oportunidad de trasladar los elementos, diseños o estructura del juego a ambientes no propiamente lúdicos como son los ambientes educativos. A esta metodología de enseñanza se la conoce como gamificación (DETERDING, 2012; WERBACH, 2014; ZAINUDDIN, 2018; ZAINUDDIN et al., 2019, 2020), y cada vez son más los estudios que muestran su cada vez más utilización en educación y sus beneficios (PARRA-GONZALEZ; SEGURA-ROBLES, 2019a).

Por otro lado, hay autores que afirman que, a través del uso de elementos del juego, como la obtención de puntos, niveles o avatares, el tiempo de dedicación de los alumnos se ve incrementado y su nivel de implicación en la realización de actividades es también mayor (ZICHERMANN; CUNNINGHAM, 2011). Por lo tanto, según estos autores, la actividad gamificada aumenta la predisposición psicológica de los alumnos para seguir en un estado activo, haciendo esto referencia al fenómeno llamado "estado de flujo" (CSIKSZENTMIHALYI, 1997), consistente en incrementar entre otras cosas, la capacidad de atención y el esfuerzo que se es capaz de dedicar a una actividad.

Una de las facilidades que se pueden encontrar en esta metodología es el uso de las Tecnologías de la Información y Comunicación. Estas van a facilitar el proceso de enseñanza y aprendizaje si se usan bien encauzadas. Para ello, los docentes tienen que aprender y experimentar con su uso, para obtener con los alumnos esos beneficios tan esperados (MARTÍNEZ CLARES; PÉREZ CUSÓ; MARTÍNEZ JUÁREZ, 2016; PARRAGONZALEZ et al., 2020; PARRA-GONZALEZ; SEGURA-ROBLES; GÓMEZ-BARAJAS, 2020; RUBILAR; ALVEAL; FUENTES, 2017). Este planteamiento metodológico innovador, pretende buscar que los estudiantes disfruten con su aprendizaje, que estén emocionados, que es la base del aprendizaje y una necesidad según muchos autores. También va a fomentar que el alumnado se implique en su aprendizaje (ALSAWAIER, 2018).

Con todo esto se refuerza la idea de que una de las principales necesidades en educación, es que los alumnos estén activos en su proceso de aprendizaje(LOPEZBELMONTE et al., 2020). Las metodologías activas, y en concreto la gamificación, fomentan todas estas necesidades que se plantean en este apartado. Por ello, la finalidad de este estudio es conocer y analizar los niveles de activación del alumnado tras la implementación de un proyecto gamificado en el aula. Además de conocer qué variables pueden estar influyendo en los resultados obtenidos o cómo se relaciona la activación con otras dimensiones como la motivación o el desarrollo del pensamiento creativo.

\section{Método}

\subsection{Participantes}

Para el estudio, tras la intervención planteada se solicitó la colaboración todos los estudiantes del curso 18/19 y 19/20 que participaron en la experiencia. En total se recibieron respuesta de 140 estudiantes de los cuales, tras la limpieza de la base de datos para eliminar datos erróneos, se obtuvo una matriz de 111 respuestas completas. Respecto al sexo de los participantes, el 61\% eran mujeres mientras que el 39\% eran hombres. Según la edad agrupada, un 41\% tenía más de 26 años mientras que un 59\% tiene 26 años o menos (Figura 1). 
Figura 1: descripción por variables de la muestra participante en el estudio.

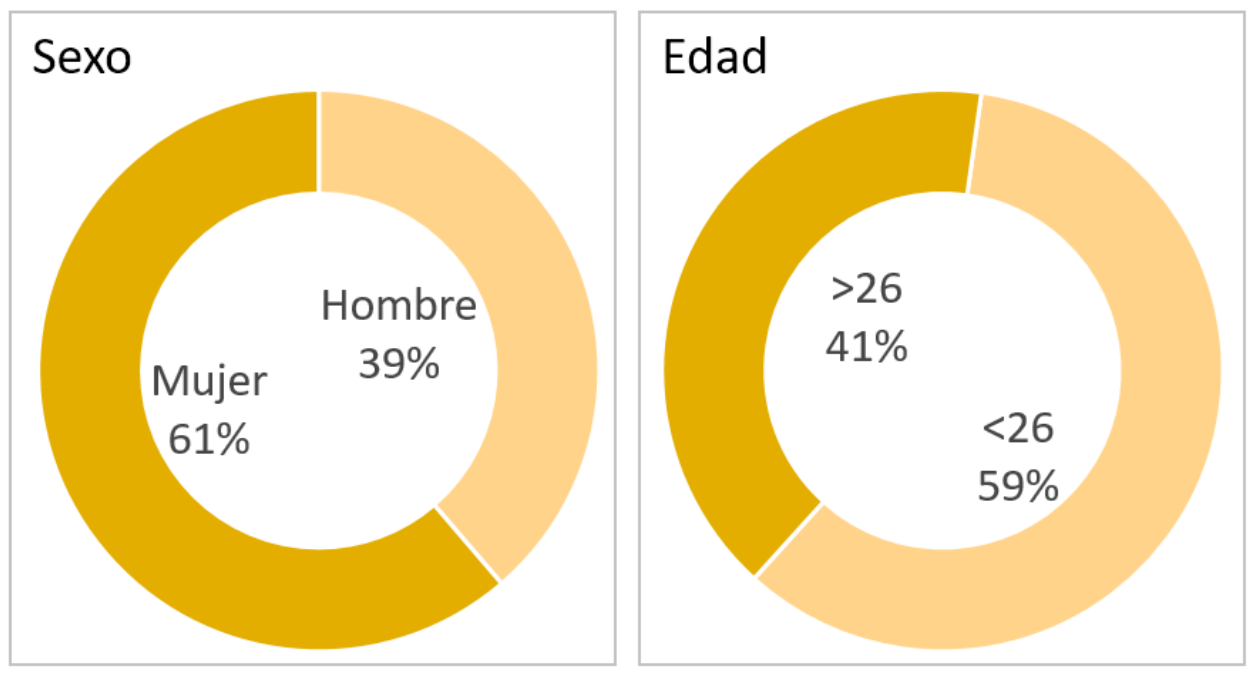

Fuente: de los autores.

\subsection{Instrumento}

Para la recogida de los datos se utilizó un cuestionario diseñado ad-hoc que recoge información en torno a distintas dimensiones. La herramienta utilizada se diseña en función de otras herramientas bien conocidas en la literatura científica (EPPMANN; BEKK; KLEIN, 2018; PARRA-GONZALEZ; SEGURA-ROBLES, 2019b). Las distintas dimensiones que componen el cuestionario general son diversión, atención, creatividad, ausencia de efecto negativo, socialización, aprendizaje, control y activación, con un total de 28 ítems. Siguiendo el propósito específico del presente artículo se utiliza la dimensión específica de activación para ser analizada en profundidad. Todas las preguntas que componen el cuestionario se evalúan en torno a una escala Likert de 5 puntos, siendo 1 el valor más negativo y 4 el más positivo.

Esta herramienta se valida en su origen desde dos perspectivas, cuantitativa y cualitativa. Para la parte cuantitativa se utiliza el alfa de Cronbach ( $\alpha$ ), estadístico ampliamente utilizado en la literatura científica (BONETT; WRIGHT, 2015; CERVANTES, 2005). Los resultados obtenidos para el mismo son de $\alpha=.701$ que pueden ser considerado aceptables. Por otro lado, desde una perspectiva cualitativa se lleva a cabo un pequeño panel Delphi para medir el grado de acuerdo o desacuerdo sobre los ítems de distintos expertos. Los resultados fueron de una percepción general positiva ( $M=4.91$; $\mathrm{SD}=0.51 ; \min =1 ; \max =5)$.

\subsection{Diseño de la propuesta}

Se llevó a cabo el trabajo con un tema que consta de 8 sesiones sobre contenidos relacionados con la asignatura a trabajar. Los profesores se encargaban de diseñar las tareas y trabajos que los alumnos tendrían que llevar a cabo de manera activa. Los profesores explicaron con anterioridad las actividades de cada sesión y la finalidad, y los alumnos tenían que realizar las tareas de manera activa, como los profesores iban indicando en las sesiones. Los profesores, además establecieron con los alumnos de 
manera activa cómo funcionarían el sistema de insignias y los rankings que se podrían desarrollar. Los contenidos se iban presentando mediante estructura y diseño de juegos, así que los estudiantes tenían que ir avanzando en la consecución de los mismos (prevaleciendo el esfuerzo empleado) para poder desarrollar los siguientes. Los estudiantes aquí adquieren todo el protagonismo ya que son los agentes principales y activos que tienen que llevar a cabo cada una de las pruebas, desafíos y retos en los sistemas de juegos del bloque temático. Las sesiones estuvieron divididas en dos bloques, de manera que en el primer bloque eran los profesores los que diseñaban las tareas de forma lúdica y en el segundo bloque, fueron los alumnos, divididos en grupos, los que se diseñaban unos grupos a otros estas tareas. En esta parte los alumnos tenían más autonomía y libertad para investigar, explorar y colaborar entre ellos para organizar las actividades de sus compañeros. En este segundo bloque tenían la experiencia de los recursos y técnicas empleados por los profesores en la primera parte y pudieron ponerlo en práctica en este.

\subsection{Procedimiento y análisis}

Los cuestionarios son recogidos a través de correo electrónico, solicitando la participación voluntaria y anónima de los participantes. La plataforma utilizada para su diseño y recogida fue Limesurvey. Una vez obtenida la matriz de datos se carga al programa de análisis estadístico SPSS. Se lleva a cabo un análisis previo para examinar su tendencia central (media, mediana) variabilidad (desviación estándar), y pico (curtosis). En la Tabla 1 se puede observar el patrón de distribución de los datos (paramétricos o no paramétricos) se observó al examinar los resultados de las estadísticas descriptivas haciendo uso de la prueba Kolmogorov-Smirnov (DODGE, 2008). El patrón de distribución de datos no sigue una distribución normal $(p<.05)$. Se hace necesario, por tanto, usar pruebas no paramétricas para realizar cualquier tipo de análisis inferencial (SHESKIN, 2011).

Tabla 1: Prueba de normalidad para la muestra utilziada.

\begin{tabular}{llll}
\hline & \multicolumn{2}{l}{ Kolmogorov-Smirnov } & $\mathrm{p}$ \\
\cline { 2 - 4 } & Estadístico & $\mathrm{gl}$ &, 000 \\
\hline Act1 &, 277 & 111 &, 000 \\
\hline Act2 &, 244 & 111 &, 000 \\
\hline Act3 &, 172 & 111 &, 000 \\
\hline Act4 &, 240 & 111 & \\
\hline
\end{tabular}

Fuente: de los autores.

En nuestro caso, recurrimos a la prueba no paramétrica $U$ de Mann-Whitney. Este tipo de pruebas, similares a la t de Student clásica, permite realizar comparaciones con grupos que nos siguen una distribución normal o que poseen menos de 50 observaciones (FÁVERO; BELFIORE, 2019; RIVAS-RUIZ; MORENO-PALACIOS; TALAVERA, 2013; SHESKIN, 2011). 


\section{Resultados}

A continuación, se presentan los resultados obtenidos en torno a las distintas variables analizadas en la presente investigación. En la Tabla 2 se presentan los descriptivos para cada uno de los ítems que componen la dimensión analizada. Así, el ítem 1 es el que mayor media obtiene (4.33; "Mientras jugaba me sentía activo") mientras que es el ítem 2 el que menores resultados obtiene (2.73; "Mientras jugaba me sentía nervioso"). Ninguno de los ítems estudiados presenta desviaciones típicas superiores a 1 , 136.

Por otro lado, las medidas de distribución nos permiten identificar la forma en que se separan o aglomeran los valores. En este sentido observamos que todos los valores de asimetría son negativos, indicando que los valores más extremos se encuentran por debajo de la media.

Las puntuaciones máximas y mínimas se sitúan en torno a 1 y 5 , lo que nos indican que no hay ningún valor extraño en la base de datos utilizada que pueda causar errores en los análisis.

Tabla 2: Estadísticos descriptivos para la dimensión activación.

\begin{tabular}{lllll}
\hline & Act1 & Act2 & Act3 & Act4 \\
\hline $\mathrm{N}$ & 111 & 111 & 111 & 111 \\
\hline Rango & 4 & 4 & 4 & 4 \\
\hline Mínimo & 1 & 1 & 1 & 1 \\
\hline Máximo & 5 & 5 & 5 & 5 \\
\hline Media & 4,33 & 2,73 & 3,32 & 4,04 \\
\hline Desv. Desviación &, 856 & 1,136 & 1,221 & 1,070 \\
\hline Varianza &, 733 & 1,290 & 1,491 & 1,144 \\
\hline Asimetría & $-1,854$ &, 589 &,- 171 &,- 1071 \\
\hline Curtosis & 4,656 & -406 &,- 964 &,- 623 \\
\hline
\end{tabular}

Fuente: de los autores.

De la misma manera, se analizan los ítems por separado y se muestra, en la Tabla 3 los resultados obtenidos para el descriptor "Act1- Mientras jugaba me sentía activo". Se muestra una concentración de respuestas en torno a los niveles 4 y 5 con un $40.5 \%$ y un $49.5 \%$ respectivamente. Siendo el 2 , como sucede en los descriptores anteriores el que menor porcentaje de respuestas obtiene (0.9\%).

Tabla 3: Estadísticos descriptivos para el ítem 1.

\begin{tabular}{llllll}
\hline & & Frecuencia & Porcentaje & \% válido & \% acumulado \\
\hline Válido & 1 & 3 & 2,7 & 2,7 & 2,7 \\
\cline { 2 - 6 } & 2 & 1 &, 9 &, 9 & 3,6 \\
\cline { 2 - 6 } & 3 & 7 & 6,3 & 6,3 & 9,9 \\
\hline & 4 & 45 & 40,5 & 40,5 & 50,5 \\
\hline & 5 & 55 & 49,5 & 49,5 & 100,0 \\
\hline
\end{tabular}

Fuente: de los autores. 
Para el descriptor "Act2- Mientras jugaba me sentía nervioso" (Tabla 4), al contrario que sucedía en el anterior, la mayoría de respuestas se acumulan entorno a los 2 y 3 con $40.5 \%$ y un $27 \%$ respectivamente. Siendo el 1 , como sucede en los descriptores anteriores el que menor porcentaje de respuestas obtiene (9.9\%).

Tabla 4: Estadísticos descriptivos para el ítem 2.

\begin{tabular}{llllll}
\hline & & Frecuencia & Porcentaje & \% válido & $\%$ acumulado \\
\hline Válido & 1 & 11 & 9,9 & 9,9 & 9,9 \\
\cline { 2 - 6 } & 2 & 45 & 40,5 & 40,5 & 50,5 \\
\cline { 2 - 6 } & 3 & 30 & 27,0 & 27,0 & 77,5 \\
\cline { 2 - 6 } & 4 & 13 & 11,7 & 11,7 & 89,2 \\
\cline { 2 - 6 } & 5 & 12 & 10,8 & 10,8 & 100,0 \\
\cline { 2 - 6 } & Total & 111 & 100,0 & 100,0 & \\
\hline
\end{tabular}

Fuente: de los autores.

Para el descriptor "Act3- Mientras jugaba me sentía frenético" (Tabla 5), al igual que con el descriptor anterior, la mayoría de respuestas se acumulan entorno a los 3 y 4 con $26.1 \%$ y un $25.2 \%$ respectivamente. Siendo el 1 , como sucede en los descriptores anteriores el que menor porcentaje de respuestas obtiene (7.2\%).

Tabla 5: Estadísticos descriptivos para el ítem 3.

\begin{tabular}{llllll}
\hline & & Frecuencia & Porcentaje & \% válido & \% acumulado \\
\hline Válido & 1 & 8 & 7,2 & 7,2 & 7,2 \\
\cline { 2 - 6 } & 2 & 23 & 20,7 & 20,7 & 27,9 \\
\cline { 2 - 6 } & 29 & 26,1 & 26,1 & 54,1 \\
\cline { 2 - 6 } & 28 & 25,2 & 25,2 & 79,3 \\
\cline { 2 - 6 } & 23 & 20,7 & 20,7 & 100,0 \\
\cline { 2 - 6 } & Total & 223 & 100,0 & 100,0 & \\
\hline
\end{tabular}

Fuente: de los autores.

Para el descriptor "Act4- Mientras jugaba me sentía emocionado" (Tabla 6), la mayoría de respuestas se acumulan entorno a los 4 y 5 con $31.5 \%$ y un $42.3 \%$ respectivamente. Siendo el 1 , como sucede en los descriptores anteriores el que menor porcentaje de respuestas obtiene (3.6\%).

Tabla 6: Estadísticos descriptivos para el ítem 4.

\begin{tabular}{llllll}
\hline & & Frecuencia & Porcentaje & \% válido & \% acumulado \\
\hline Válido & 1 & 4 & 3,6 & 3,6 & 3,6 \\
\cline { 2 - 6 } & 2 & 6 & 5,4 & 5,4 & 9,0 \\
\cline { 2 - 6 } & 3 & 19 & 17,1 & 17,1 & 26,1 \\
\cline { 2 - 6 } & 4 & 35 & 31,5 & 31,5 & 57,7 \\
\hline
\end{tabular}


Belo Horizonte, v. 13, n. 3, p. 278-293, set.-dez. 2020 - ISSN 1983-3652 DOI : $10.35699 / 1983-3652.2020 .25846$

\begin{tabular}{lllll}
\hline 5 & 47 & 42,3 & 42,3 & 100,0 \\
\cline { 2 - 5 } & Total & 223 & 100,0 & 100,0 \\
\hline
\end{tabular}

Fuente: de los autores.

Para observas estas distinciones se presentan los resultados de medias y desviación típica de forma gráfica. Así, de la misma manera, se observa la prevalencia de respuestas altas en contraposición de aquellas respuestas de menor valor, además los bigotes en las gráficas muestran que las desviaciones típicas en las respuestas de los participantes no son altas (Figura 2).

Figura 2: Gráficos de frecuencias de los ítems analizados.

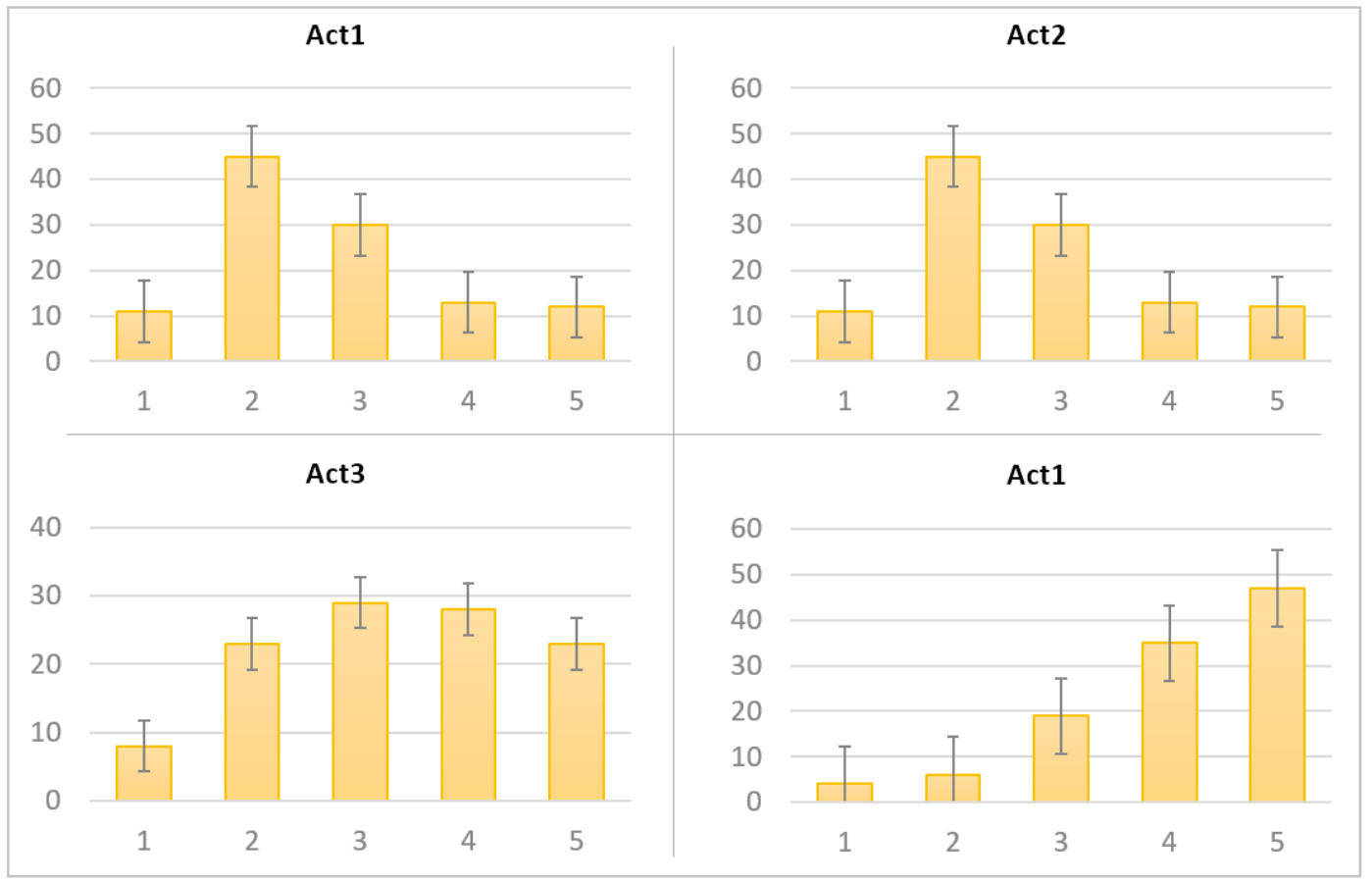

Fuente: de los autores.

\subsection{Análisis inferenciales}

Del mismo modo y, tras el estudio descriptivo realizado en el apartado anterior, se procede a realizar distintos análisis inferenciales con el objetivo de analizar la influencia de las distintas variables sociodemográficas recogidas durante el estudio y conocer cómo afectas estas a las respuestas de los participantes.

Respecto al sexo, los resultados (Tabla 7) no muestran diferencias significativas para ninguno de los Act1 $(p=.648)$, Act2 $(p=.255)$ y Act3 $(p=.209)$, indicando que el sexo no parece ser una variable diferenciadora en torno a la activación de los participantes en actividades gamificadas. No ocurre lo mismo en el ítem 4, donde aparece una diferencia significativa $(p=.039)$. Son las mujeres las que obtienen valores más alto que los hombres. 
Belo Horizonte, v. 13, n. 3, p. 278-293, set.-dez. 2020 - ISSN 1983-3652 DOI : $10.35699 / 1983-3652.2020 .25846$

Tabla 7: Diferencia en los valores de activación según el género.

\begin{tabular}{|c|c|c|c|c|c|c|}
\hline \multicolumn{7}{|c|}{ Rangos } \\
\hline & Genero & $\mathrm{N}$ & & \multicolumn{2}{|c|}{ Rango promedio } & Suma de rangos \\
\hline \multirow{2}{*}{ Act1 } & Hombre & \multicolumn{2}{|l|}{43} & \multicolumn{2}{|c|}{54,42} & 2340,00 \\
\hline & Mujer & \multicolumn{2}{|l|}{68} & 57,00 & & 3876,00 \\
\hline \multirow{2}{*}{ Act2 } & Hombre & \multicolumn{2}{|l|}{43} & 51,83 & & 2228,50 \\
\hline & Mujer & \multicolumn{2}{|l|}{68} & 58,64 & & 3987,50 \\
\hline \multirow{2}{*}{ Act3 } & Hombre & \multicolumn{2}{|l|}{43} & 52,06 & & 2238,50 \\
\hline & Mujer & \multicolumn{2}{|l|}{68} & 58,49 & & 3977,50 \\
\hline \multirow{2}{*}{ Act4 } & Hombre & 43 & & 63,48 & & 2729,50 \\
\hline & Mujer & \multicolumn{2}{|l|}{68} & 51,27 & & 3486,50 \\
\hline \multicolumn{7}{|c|}{ Estadísticos de la prueba } \\
\hline & & & Act1 & Act2 & Act3 & Act4 \\
\hline \multicolumn{2}{|c|}{ U de Mann-Whitney } & & 1394,000 & 1282,500 & 1292,500 & 1140,500 \\
\hline \multicolumn{2}{|c|}{ W de Wilcoxon } & & 2340,000 & 2228,500 & 2238,500 & 3486,500 \\
\hline \multicolumn{2}{|c|}{ Z } & &,- 457 & $-1,139$ & $-1,054$ & $-2,066$ \\
\hline \multicolumn{2}{|l|}{ Sig. } & & ,648 & 255 & 292 &, 039 \\
\hline
\end{tabular}

Fuente: de los autores.

Desde otra perspectiva, se realizan las pruebas inferenciales para la variable edad, que ha sido dicotomizada en dos bloques (Tabla 8), por un lado, quienes tienen 260 menos años y, por otro, quienes tienen más de 26 años. Los resultados no muestran diferencias significativas respecto a esta variable en los ítems $2(p=.504)$ y $4(p=.359)$. Por el contrario, los ítems 2 y 3 sí muestran diferencias respecto a la edad de los participantes con $p=.27$ y $p=.005$ respectivamente. En ambos casos los que mayores valores obtienen son los menores de 26.

Tabla 8: Diferencia en los valores de activación según la edad.

\begin{tabular}{|c|c|c|c|c|c|}
\hline \multicolumn{6}{|c|}{ Rangos } \\
\hline & Edad & $\mathrm{N}$ & $\mathrm{RP}^{*}$ & \multicolumn{2}{|c|}{$\mathrm{SR}^{\star \star}$} \\
\hline \multirow{2}{*}{ Act1 } & $=<26$ & 66 & 50,97 & \multicolumn{2}{|c|}{3364,00} \\
\hline & $>26$ & 45 & 63,38 & \multicolumn{2}{|c|}{2852,00} \\
\hline \multirow{2}{*}{ Act2 } & $=<26$ & 66 & 54,39 & \multicolumn{2}{|c|}{3590,00} \\
\hline & $>26$ & 45 & 58,36 & \multicolumn{2}{|c|}{2626,00} \\
\hline \multirow{2}{*}{ Act3 } & $=<26$ & 66 & 49,03 & \multicolumn{2}{|c|}{3236,00} \\
\hline & $>26$ & 45 & 66,22 & \multicolumn{2}{|c|}{2980,00} \\
\hline \multirow{2}{*}{ Act4 } & $=<26$ & 66 & 58,18 & \multicolumn{2}{|c|}{3840,00} \\
\hline & $>26$ & 45 & 52,80 & \multicolumn{2}{|c|}{2376,00} \\
\hline \multicolumn{6}{|c|}{ Estadísticos de prueba } \\
\hline & & Act1 & Act2 & Act3 & Act4 \\
\hline \multicolumn{2}{|c|}{ U de Mann-Whitney } & 1153,000 & 1379,000 & 1025,000 & 1341,000 \\
\hline \multicolumn{2}{|c|}{ W de Wilcoxon } & 3364,000 & 3590,000 & 3236,000 & 2376,000 \\
\hline
\end{tabular}


Belo Horizonte, v. 13, n. 3, p. 278-293, set.-dez. 2020 - ISSN 1983-3652 DOI : $10.35699 / 1983-3652.2020 .25846$

\begin{tabular}{lcccc}
\hline Z & $-2,214$ &,- 667 & $-2,838$ &,- 918 \\
\hline Sig. &, 027 &, 504 &, 005 &, 359 \\
\hline \multicolumn{4}{c}{ * Rangos promedios **Suma de Rangos. }
\end{tabular}

Fuente: de los autores.

Por último, es interesante conocer cómo se relaciona la activación con otras dimensiones analizadas. Para este caso, se calcula el sumativo total de la dimensión para facilitar la comparación con otras dimensiones. Como se observa en la Tabla 9 se encuentran correlaciones positivas para todas las dimensiones comparadas. La activación muestra valores altos tanto para el pensamiento creativo $(r=, 460 ; p<0,01)$ como para la motivación $(r=, 392 ; p<0,01)$.

Tabla 9: Correlaciones para la dimensión activación, motivación y pensamiento creativo.

\begin{tabular}{|c|c|c|c|c|}
\hline & & Activación & Motivación & Pensamiento Creativo \\
\hline \multirow[b]{2}{*}{ Activación } & Correlación & 1 &, $392^{\star \star}$ &, $460 * \star$ \\
\hline & Sig. & & ,000 & ,000 \\
\hline \multirow{2}{*}{ Motivación } & Correlación &, $392^{\star \star}$ & 1 &, $592^{\star *}$ \\
\hline & Sig & ,000 & & ,000 \\
\hline \multirow{2}{*}{$\begin{array}{l}\text { Pensamiento } \\
\text { Creativo }\end{array}$} & $r$ &, 460 ** &, $592^{\star \star}$ & 1 \\
\hline & Sig & ,000 & ,000 & \\
\hline
\end{tabular}

\section{Discussión}

Como se ha evidenciado en el apartado anterior, esta investigación tiene hallazgos interesantes para analizar desde el punto de vista educativo, para que los docentes los tengan en cuenta, de cara a seleccionar una metodología de enseñanza, dado que actualmente los propios docentes buscan renovaciones metodológicas que satisfagan sus necesidades y las de sus alumnos, así como obtener distintos beneficios mediante las mismas. Algo que han mostrado otras investigaciones similares (ALSAWAIER, 2018; BERKLING; THOMAS, 2013; BURKE, 2014).

Se ha encontrado que los estudiantes se sienten activos en su mayoría durante el proceso de enseñanza-aprendizaje; esto es un elemento esencial para el aprendizaje, ya que los estudiantes cuando están activos, son los protagonistas activos que construyen su aprendizaje (GAYÁ, 2016; HINOJO-LUCENA et al., 2018; LÓPEZ-GUEDE, 2015; VARGAS, 2006).

Por otro lado, los alumnos han indicado con valores entre 3 y 4 que se sentían nerviosos mientas jugaban, como en otros estudios sobre gamificación (RODRÍGUEZ HERRERA et al., 2019). En relación a este nivel de "nerviosismo" mientras se realiza la actividad, hay que intentar que el mismo sea positivo, no siendo este un nerviosismo con efecto negativo, ya que eso puede provocar rechazo por parte de los alumnos a la hora de 
afrontar una actividad y llegar incluso a verla de manera negativa y con ansiedad, que es lo contrario de lo que se pretende mediante las metodologías activas, que se pretende motivar al alumnado y mantenerlos activos para fomentar y mejorar su aprendizaje (LOPEZ-QUINTERO; PONTES-PEDRAJAS; VARO-MARTÍNEZ, 2019; ROBSON et al., 2015).

Al igual que en la variable anterior, y en las mismas cantidades, los alumnos manifiestan que mientras jugaban se sentían frenéticos, hay que tener en cuenta, igual que en el ítem anterior, que ese frenetismo sea positivo, y no caiga en lo negativo, para evitar caer en las desventajas, como hemos dicho anteriormente, para ello se utilizarán estrategias de motivación positivas (BARCA-LOZANO et al., 2012), que repercutan positivamente en el aprendizaje.

Por otro lado, la mayoría de los alumnos han puntuado entre 4 y 5 que mientras jugaban se sentían emocionados, lo cual es clave para el aprendizaje. Son muchos los estudios que defienden que las personas aprenden cuando se emocionan (GARCíARETANA, 2012) con lo que los docentes deberían intentar que los alumnos se emocionen para fomentar y favorecer el aprendizaje desde este punto de vista.

La relación que mantienen los distintos factores cognitivos, sobre todo con el logro o las puntuaciones obtenidas por los estudiantes ha sido ampliamente estudiado en la literatura, sobre la relación motivación-logro (ALDERMAN, 2008; BUSATO et al., 2000; YILDIRIM, 2017). Igualmente, la activación ha demostrado ser un aspecto esencial en la mayoría de experiencias educativas que se llevan a cabo hoy en día (CASSIDY, 2004). Nuestros resultados también indican que los participantes han aumentado sus niveles de activación al verse inmerso en el desarrollo dela experiencia, no encontrándose diferencias significativas importantes de manera generalizada.

Aunque los procesos gamificados están ganando mucho peso en los sistemas actuales se puede apreciar una falta importante de investigaciones que relacionen sus efectos con el desarrollo de la creatividad o el pensamiento creativo (KALINAUSKAS, 2014; ROMERO-RODRÍGUEZ; TORRES-TOUKOUMIDIS; AGUADED, 2016).

\section{Conclusiones}

Estos hallazgos son interesantes para la comunidad científica en general y los docentes en particular, ya que el uso de esta metodología promueve beneficios que los propios docentes buscan en el aprendizaje de sus alumnos. Viendo estos resultados, en línea con estas investigaciones, paree ser una buena metodología la gamificación para fomentar el aprendizaje

Las metodologías activas, en concreto las experiencias gamificadas, no dejan de ser una herramienta que, en mayor o menor medida, mejora distintos procesos psicológicos relacionados con el aprendizaje. Esta mejora, acaban influyendo, en la mayoría de ocasiones en los resultados que obtienen los estudiantes 0 en sus capacidades, como el desarrollo del pensamiento creativo. 


\section{Consideraciones finales y prospectivas}

Aunque el estudio analiza cómo afectan la experiencia gamifcada desarrollada a los niveles de activación de los estudiantes es necesario, en el ámbito científico, abrir nuevas vías y propuestas para investigaciones futuras. Entre las posibles investigaciones a desarrollar en adelante se proponen las siguientes líneas:

- Seleccionar una muestra de un ámbito distinto al universitario, por ejemplo, con estudiantes de Educación Secundaria Obligatoria.

- Comparar si éstos niveles de activación difieren dependiendo del ámbito universitario donde se desarrollan.

- Comprobar si estos niveles de activación son distintos entre los docentes y los estudiantes y si existen diferencias significativas.

- Detectar qué variables sociodemográficas pueden influir en estos niveles más allá del género.

\section{Referencias}

ALDERMAN, M. K. Motivation for achievement: possibilities for teaching and learning. New York, NY: Routledge, 2008.

ALSAWAIER, R. S. The Effect of Gamification on Motivation and Engagement. International Journal of Information and Learning Technology, v. 35, n. 1, p. 56-79, 2 jan. 2018.

BARCA-LOZANO, A.; ALMEIDA, L. S.; PORTO-RIOBOO, A. M.; PERALBO-UZQUIANO; M.; BRENLLA-BLANC. J. C. Motivación escolar y rendimiento: impacto de metas académicas, de estrategias de aprendizaje y autoeficacia. Anales de psicología, v. 28, n. 3, p. 848-859, 2012. Disponible en: https://core.ac.uk/download/pdf/61918873.pdf. Acceso en: 15 oct. 2020.

BENITEZ-MURILLO, M. I. E Juego como Herramienta de Aprendizaje. Innovación y Experiencias Educativas, n. 16, 2009. Disponible en: https://archivos.csif.es/archivos/andalucia/ensenanza/revistas/csicsif/revista/pdf/ Numero 16/MARIA\%20ISABEL_BENITEZ_1.pdf. Acceso en: 15 oct. 2020.

BERKLING, K.; THOMAS, C. Gamification of a Software Engineering course and a detailed analysis of the factors that lead to it's failure. 2013. International Conference on Interactive Collaborative Learning (ICL). Anais... Kazan, Russia: IEEE, set. 2013. Disponible en: http://ieeexplore.ieee.org/document/6644642/. Acceso en: 17 mar. 2020.

BONETT, D. G.; WRIGHT, T. A. Cronbach's alpha reliability: Interval estimation, hypothesis testing, and sample size planning. Journal of Organizational Behavior, v. 36, n. 1, p. 3-15, jan. 2015.

BURKE, B. Gamify: How Gamification Motivates People to Do Extraordinary Things. 
Brookline, MA: Bibliomotion, books + media, 2014.

BUSATO, V. V.; PRINS, F. J.; JELSHOUT, J.; HAMAKER, C. Intellectual ability, learning style, personality, achievement motivation and academic success of psychology students in higher education. Personality and Individual Differences, v. 29, n. 6, p. 1057-1068, dez. 2000. https://doi.org/10.1016/S0191-8869(99)00253-6

CASSIDY, S. Learning Styles: An overview of theories, models, and measures. Educational Psychology, v. 24, n. 4, p. 419-444, ago. 2004.

CERVANTES, V. Interpretaciones del coeficiente alpha de Cronbach. Avances en medición, v. 3, n. 1, p. 9-28, 2005.

CSIKSZENTMIHALYI, M. Finding flow: the psychology of engagement with everyday life. 1st ed. New York, NY: Basic Books, 1997.

DETERDING, S. Gamification: designing for motivation. interactions, v. 19, n. 4, p. 14, jul. 2012.

DODGE, Y. The concise encyclopedia of statistics. 1st. ed. New York: Springer, 2008.

EPPMANN, R.; BEKK, M.; KLEIN, K. Gameful Experience in Gamification: Construction and Validation of a Gameful Experience Scale [GAMEX]. Journal of Interactive Marketing, v. 43, p. 98-115, ago. 2018.

FÁVERO, L. P.; BELFIORE, P. Univariate Descriptive Statistics. In: Data Science for Business and Decision Making. London: Academic Press, 2019. p. 21-91.

FERNANDEZ-MARCH, A. Metodologías activas para la formación de competencias. Educatio Siglo XXI, v. 24, n. 1, p. 35-56, 2006.

FOSTER, A. Games and Motivation to Learn Science: Personal Identity, Applicability, Relevance and Meaningfulness. Journal of Interactive Learning Research, v. 19, n. 4, p. 597-614, out. 2008.

GARCÍA-RETANA, J. Á. La educación emocional, su importancia en el proceso de aprendizaje. Revista Educación, v. 36, n. 1, p. 1-24, 2012.

GAYÁ, V. ENSEÑAR A PENSAR: Las nuevas metodologías colocan al alumno como parte activa de su propio aprendizaje. El siglo de Europa, n. 1177, p. 36-37, 2016.

HINOJO-LUCENA, F.; MINGORANCE-ESTRADA, A. C.; TRUJILLO-TORRES, T. M.; AZNAR-DÍAZ, I.; RECHE, M. P. C. Incidence of the Flipped Classroom in the Physical Education Students' Academic Performance in University Contexts. Sustainability, v. 10, n. 5, p. 13-34, 25 abr. 2018. https://doi.org/10.3390/su10051334

KALINAUSKAS, M. Gamification in Fostering Creativity. Social Technologies, v. 4, n. 1, p. 
62-75, 2014.

LABRADOR PIQUER, M. J.; ANDREU ANDRÉS, M. A. Metodologías activas. Valencia: Universidad Politécnica de Valencia, 2008.

LIRA VALDIVIA, R. I. Las metodologías activas y el foro presencial: su contribución al desarrollo del pensamiento crítico. Revista Electrónica "Actualidades Investigativas en Educación", v. 10, n. 1, p. 1-18, 2010.

LOPEZ-BELMONTE, J. et al. Evaluating Activation and Absence of Negative Effect: Gamification and Escape Rooms for Learning. International Journal of Environmental Research and Public Health, v. 17, n. 7, p. 22-24, 26 mar. 2020.

LÓPEZ-GUEDE, J. M. L. Experiencia docente mediante la metodología de Aprendizaje Basado en Problemas. Ikastorratza, e-Revista de didáctica, n. 14, p. 5, 2015.

LOPEZ-QUINTERO, J. L.; PONTES-PEDRAJAS, A.; VARO-MARTÍNEZ, M. Las TIC en la enseñanza científico-técnica hispanoamericana: Una revisión bibliográfica. Digital Education Review, v. 0, n. 35, p. 229-243, 2019.

LUELMO DEL CASTILLO, M. J. Origen y desarrollo de las metodologías activas dentro del sistema educativo español. Encuentro: revista de investigación e innovación en la clase de idiomas, n. 27, p. 4-21, 2018.

MARTÍNEZ CLARES, P.; PÉREZ CUSÓ, J.; MARTÍNEZ JUÁREZ, M. Las TICs y el entorno virtual para la tutoría universitaria. Educación XX1, v. 19, n. 1, p. 287-310, 11 nov. 2016.

MONTAÑÉS RODRÍGUEZ, J.; CASADO, M. P.; SÁNCHEZ, T.; LÓPEZ, R.; POSTIGO, J. M. L.; PORTAS, PILAR BLANC; SÁNCHEZ, M. J.; SERRANO, J. P., MORATALLA, P. T. EI juego en el medio escolar. Ensayos: Revista de la Facultad de Educación de Albacete, $\mathrm{n}$. 15 , p. 235-260, 2000.

NEVES, A. de J. Protagonistas de Nuestro Aprendizaje. Edetania. Estudios y propuestas socioeducativas, v. 1, n. 44, p. 231-240, dec. 2013.

PARRA-GONZALEZ, M. E.; BELMONTE, J. L.; SEGURA-ROBLES, A.; CABRERA, A. F. Active and Emerging Methodologies for Ubiquitous Education: Potentials of Flipped Learning and Gamification. Sustainability, v. 12, n. 2, p. 1-11, 14 jan. 2020. https://doi.org/10.3390/su12020602

PARRA-GONZALEZ, M. E.; SEGURA-ROBLES, A. Producción científica sobre gamificación en educación: un análisis cienciométrico. [Scientific production on gamification in education: a scientometric analysis]. Revista de Educación, v. 5, n. 386, p. 113-131, 2019a.

PARRA-GONZALEZ, M. E.; SEGURA-ROBLES, A. Traducción y Validación de la Escala 
de Evaluación de Experiencias Gamificadas (GAMEX). Bordón. Revista de Pedagogía, v. 1, n. 1, p. 87-99, 12 set. 2019 b.

PARRA-GONZALEZ, M. E.; SEGURA-ROBLES, A.; GÓMEZ-BARAJAS, E. R. Assessing Gamified Experiences in Physical Education Teachers and Students. IJERI: International Journal of Educational Research and Innovation, v. 1, n. 13, p. 166-176, 4 fev. 2020.

PIAGET, J. Play, dreams and imitation in childhood. 3. ed. London: Routledge, 2013.

RIVAS-RUIZ, R.; MORENO-PALACIOS, J.; TALAVERA, J. O. Diferencias de medianas con la U de Mann-Whitney. Rev. Med. Inst. Mex .Seguro Soc., v. 51, n. 4, p. 414-419, 2013.

ROBSON, K.; PLANGGER, K.; KIETZMANN, J. H.; CARTHY, I. Mc.; PITT, L. Is it all a game? Understanding the principles of gamification. Business Horizons, v. 58, n. 4, p. 411420, jul. 2015. https://doi.org/10.1016/j.bushor.2015.03.006

RODRÍGUEZ-PÉREZ, N. Causas que intervienen en la motivación del alumno en la enseñanza-aprendizaje de idiomas: el pensamiento del profesor. Didáctica. Lengua y Literatura, v. 24, n. 0, p. 359-387, 27 set. 2012.

ROMERO-RODRÍGUEZ, L.-M.; TORRES-TOUKOUMIDIS, Á.; AGUADED, I. Ludificación y educación para la ciudadanía. Revisión de las experiencias significativas. Educar, v. 53, n. 1, p. 109, 23 dez. 2016.

RUBILAR, P. S.; ALVEAL, F. R.; FUENTES, A. C. M. Evaluación de la alfabetización digital y pedagógica en TIC, a partir de las opiniones de estudiantes en Formación Inicial Docente. Educação e Pesquisa, v. 43, n. 1, p. 127-143, mar. 2017.

SEGURA-ROBlES, A.; FUENTES-CABRERA, A., PARRA-GONZÁLEZ, M. E.; LÓPEZBELMONTE, J. Effects on Personal Factors Through Flipped Learning and Gamification as Combined Methodologies in Secondary Education. Frontiers in Psychology, v. 11, p. 1-8, jun. 2020. https://doi.org/10.3389/fpsyg.2020.01103

SHESKIN, D. Handbook of parametric and nonparametric statistical procedures. 5th ed. Boca Raton: CRC Press, 2011.

VARGAS, E. D. La situación de enseñanza y aprendizaje como sistema de actividad: el alumno, el espacio de interacción y el profesor. Revista Iberoamericana de Educación, v. 39, n. 4, p. 1-11, 2006.

WERBACH, K. (Re)Defining Gamification: A Process Approach. In: SPAGNOLLI, A.; CHITTARO, L.; GAMBERINI, L. (EDS.). Persuasive Technology. Anais...: Lecture Notes in Computer Science.Cham: Springer International Publishing, 2014. p 266-272.

YILDIRIM, I. The effects of gamification-based teaching practices on student achievement and students' attitudes toward lessons. The Internet and Higher Education, v. 33, p. 86-92, 
abr. 2017.

ZAINUDDIN, Z. Students' learning performance and perceived motivation in gamified flipped-class instruction. Computers \& Education, v. 126, p. 75-88, nov. 2018.

ZAINUDDIN, Z. et al. How do students become self-directed learners in the EFL flippedclass pedagogy? A study in higher education. Indonesian Journal of Applied Linguistics, $\mathrm{V}$. 8, n. 3, p. 678-690, 2019.

ZAINUDDIN, Z.; WAHCHU, S. K.; SHUJAHAT, M.; PERERA, C. J. The impact of gamification on learning and instruction: A systematic review of empirical evidence. Educational Research Review, v. 30, p. 100-326, jun. 2020. https://doi.org/10.1016/j.edurev.2020.100326

ZICHERMANN, G.; CUNNINGHAM, C. Gamification by design: implementing game mechanics in web and mobile apps. 1st. ed. Sebastopol, Calif: O'Reilly Media, 2011.

Recebido em dia 23 de junho de 2020. Aprovado em dia 29 de julho de 2020. 